\title{
Acute Leukemia and Severe Thrombocytopenia in Neurosurgical Patient: A Case Report
}

\author{
Yao Christian Hugues Dokponou ${ }^{1,2^{*}} \mathbb{D}^{\mathbb{D}}$, Fernand Nathan Imoumby ${ }^{1,2}$, Napoléon Imbunhe1,2, \\ Miloud Gazzaz1,2 \\ ${ }^{1}$ Mohammed V Military Teaching Hospital, Rabat, Morocco \\ ${ }^{2}$ Faculty of Medicine and Pharmacy, Mohammed V University, Rabat, Morocco \\ Email: ^dokponou2407@gmail.com, ifernandnathan@gmail.com, imbunhe@gmail.com, gazzazmiloudi@hotmail.com
}

How to cite this paper: Dokponou, Y.C.H., Imoumby, F.N., Imbunhe, N. and Gazzaz, M. (2021) Acute Leukemia and Severe Thrombocytopenia in Neurosurgical Patient: A Case Report. Open Access Library Journal, 8: e7516.

https://doi.org/10.4236/oalib.1107516

Received: May 12, 2021

Accepted: July 25, 2021

Published: July 28, 2021

Copyright $\odot 2021$ by author(s) and Open Access Library Inc.

This work is licensed under the Creative Commons Attribution International License (CC BY 4.0).

http://creativecommons.org/licenses/by/4.0/

\begin{abstract}
Severe thrombocytopenia (less than 50,000 platelets count per microliter with the normal range of 150,000 to 450,000 per microliter) can complicate the management of neurosurgical patients by significantly increasing the potential risk of bleeding during or after invasive procedures. Even though the current standard-of-care treatment for severe thrombocytopenia is platelet transfusion, the optimal platelet count for safe neurosurgical procedure is still controversial. We report a case of a 57-year-old woman, newly diagnosed with acute leukemia, and followed up in the clinical hematology department that is a candidate for external ventricular shunt (EVS) for acute hydrocephalus revealed by intracranial hypertension and drowsiness. Severe thrombocytopenia was found in her laboratory result of blood analysis. She had been reoperated the same day for acute subdural hematoma as a complication of the drainage. The patient died of septic shock in the ICU two weeks later.
\end{abstract}

\section{Subject Areas}

Neurology

\section{Keywords}

External Ventricular Shunt, Acute Subdural Hematoma, Acute Leukemia, Severe Thrombocytopenia, Case Report

\section{Introduction}

Achieving and maintaining hemostasis in neurosurgical procedures is critical to the outcome [1]. Most studies examining the prognostic value of preoperative coagulation testing are too small to examine the predictive value of routine 
preoperative coagulation testing in patients having noncardiac surgery. Platelet count abnormalities found in the course of routine preoperative screening are associated with a higher risk of blood transfusion and death [2]. On the other hand, hydrocephalus is a frequent entity in neurosurgical practice, the most common etiologies of which are represented by obstruction to the normal flow of CSF in the presence of a solid tumor or an alteration in the resorption of the CSF in the case of subarachnoid hemorrhage or anterior meningitis. Hydrocephalus can be found in patients with leukemia as previously reported in autopsy studies [3] [4]. We report the exceptional case of a 57-year-old female patient, newly diagnosed with acute leukemia under chemotherapy, who underwent surgery for external ventricular shunt (EVS) in the context of severe thrombocytopenia, for the management of acute hydrocephalus complicated by acute subdural hematoma.

\section{Case Presentation}

A 57-year-old female patient with a past medical history of hypertension and newly diagnosed for acute leukemia under chemotherapy underwent surgery for external ventricular shunt (EVS) to manage acute hydrocephalus complicated by acute subdural hematoma.

The patient was diagnosed with acute hydrocephalus revealed by intracranial hypertension and drowsiness with a GCS (Glasgow Coma Scale) of 14 (Table 1). The patient had severe thrombocytopenia (platelet count $28 \times 10^{9} / \mathrm{L}$ ). We decided to wait for the surgical procedure to allow the clinical hematology colleagues to raise the platelet count to a reasonable one that can be suitable for surgery by platelet transfusion. The next day the platelet count rises to $48 \times$ $10^{9} / \mathrm{L}$. She lost two (2) points of her GCS with the persistence of quadriventricular dilatation on the CT-scan (Figure 1). The decision to operate in such a condition was not easy to make, but since her GCS is now about 12, we took her to the operating room for EVS. Immediately after the shunt, she did well and became alert with a GCS of 15.

Eight hours later, the EVS brought about $120 \mathrm{ml}$, and the patient was unconscious GCS 8. We rushed her to the CT-Scan and got an acute right-hemispheric subdural hematoma (Figure 2). The platelet count is now $57 \times 10^{9} / \mathrm{L}$ ). No other

Table 1. Glasgow coma scale (GCS).

\begin{tabular}{ccc}
\hline Eye opening & Verbal response & Motor response \\
\hline $1=$ None & $1=$ None & 1 = None \\
$2=$ To pain & $2=$ Incomprehensive sounds & $2=$ Abnormal extension \\
$3=$ To voice & $3=$ Inappropriate words & $3=$ Abnormal flexion \\
$4=$ Spontaneously & $4=$ Confused & $4=$ Withdraws to pain \\
& $5=$ Oriented & $5=$ Localises to pain \\
& & $6=$ Obeys commands
\end{tabular}




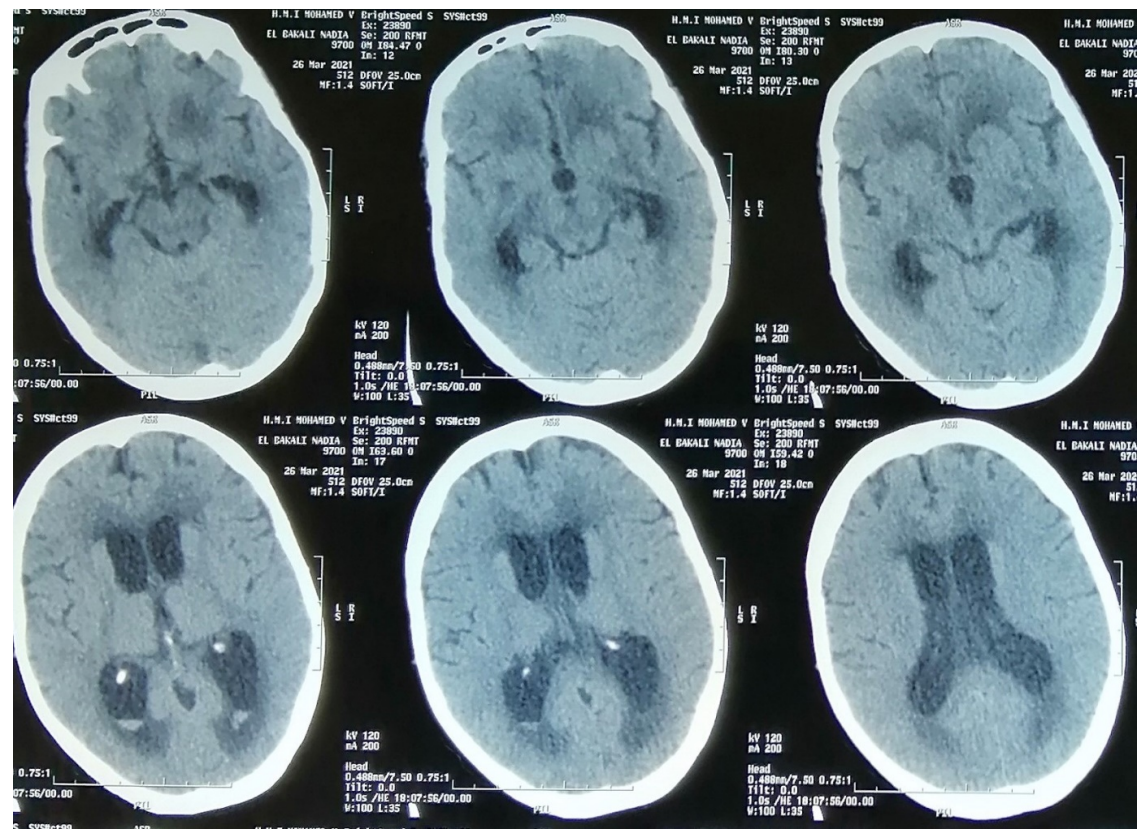

Figure 1. Brain CT-scan, parenchymal window, showing dilatation of the four ventricles with an intraventricular bleed, slight brain edema.

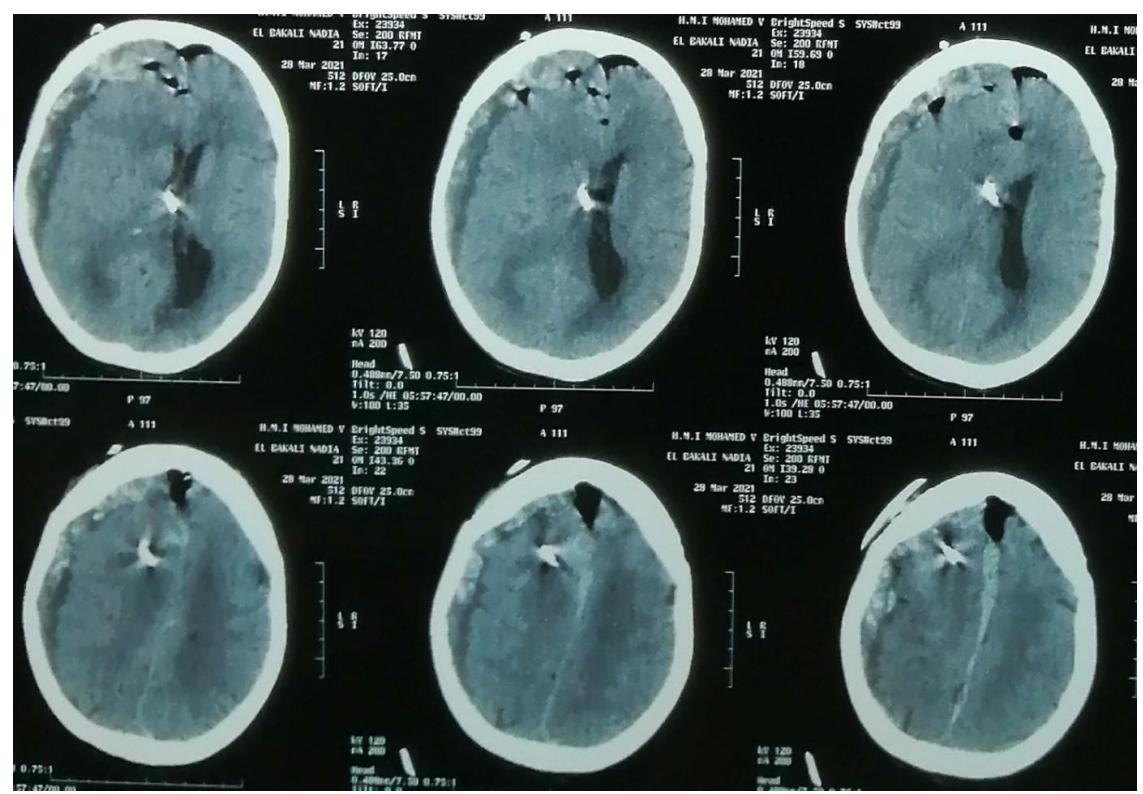

Figure 2. Appearance of acute subdural right hemispheric hematoma $7 \mathrm{H}$ after the external ventricular shunt, on a brain CT-scan.

obvious abnormality. The patient underwent surgery to evacuate the hematoma by craniectomy (Figure 3). Standard anesthesia and intraoperative monitoring regimen were deployed, and the surgical procedure was uneventful. She was then admitted to the ICU. She received an infusion of intravenous fluids and correction of metabolic acidosis; no rise of intracranial pressure or excessive surgical bleeding was seen. The hematoma was well evacuated but the patient died two weeks later for septic choc. 

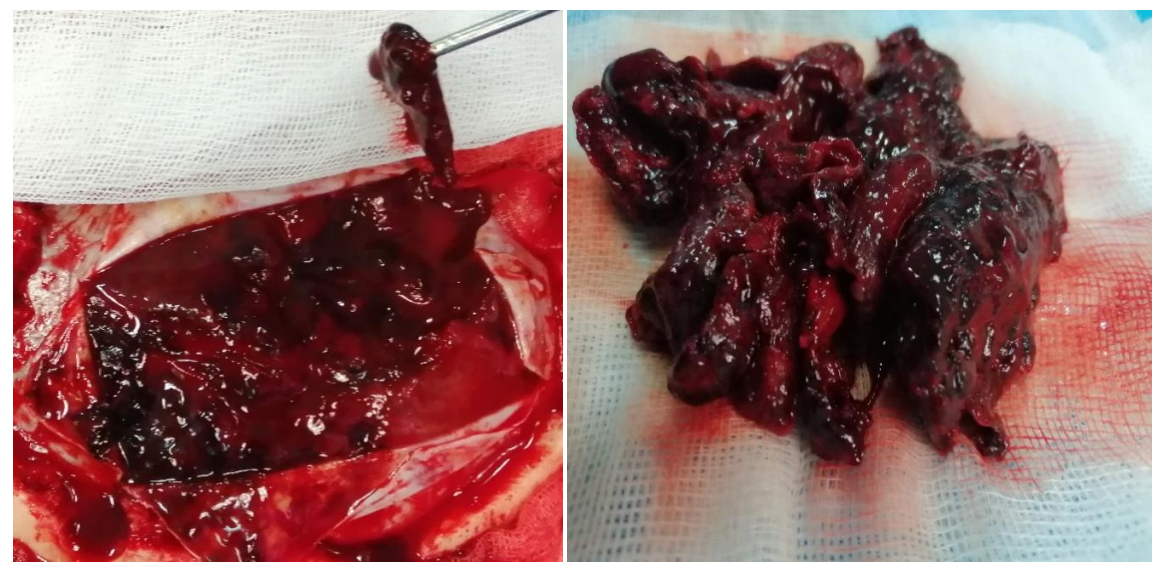

Figure 3. Evacuation of the hematoma.

\section{Discussion}

Our patient was newly diagnosed with acute leukemia. When people have Acute myeloid leukemia, blasts make copies of themselves quickly. This slows the production of red blood cells and platelets, causing tiredness from anemia and a risk of bleeding from a low platelet count [5]. It may be common to associate acute leukemia with thrombocytopenia as reported in several papers [6] [7]. Our patient received chemotherapy, and it is known that coagulation homeostasis may become further impaired after nonsurgical cancer therapy, especially after preoperative irradiation, which produces lesions precipitating both bleeding and thrombosis. Anticancer chemotherapy may affect liver function and decrease the synthesis of both pro coagulation and anticoagulation factors. Most chemotherapeutic protocols affect platelet synthesis, which arises from a principal dose-limiting side effect [8]. The patient was diagnosed with acute hydrocephalus revealed by intracranial hypertension and drowsiness with a GCS of 14. In the previous autopsy studies [3] [4], a high degree of correlation was found between hydrocephalus and meningeal leukemia syndrome. Hydrocephalus was thought to be due probably to leukemic infiltrations within the arachnoid which interfered with cerebrospinal fluid flow and subsequently resulted in increased intracranial pressure. In those series, acute hydrocephalus was not observed, which refers to a mechanism either unknown or to acute hydrocephalus secondary to unnoticed subarachnoid hemorrhage caused by the severe thrombocytopenia.

We decided to wait for the surgical procedure until the level of the platelet count raised to a reasonable one that can be suitable for surgery. In a large observational study of more than 300,000 noncardiac surgical patients without known indications for preoperative coagulation testing, Glance et al. [2] found that patients with moderate-to-severe thrombocytopenia had a 75\% higher risk of receiving a blood transfusion, and a $90 \%$ higher risk of 30-day mortality, after adjusting for preoperative risk and surgical complexity. This is a fact even if several commentators have advised that routine preoperative testing is unnecessary 
[9]. According to Schramm et al. [10], coagulation studies are ordered routinely as part of the preoperative evaluation of neurosurgical patients in their hospital. Reasons for this practice include: 1) lack of evidence that omitting coagulation tests in neurosurgical patients is safe; 2) fear of the consequences of postoperative bleeding following neurosurgery; and 3) suspicion that coagulation disorders and thrombocytopenia may occur more frequently in neurosurgical patients. The patient's neurological condition continued to deteriorate, prompting us to make the decision to operate despite an unsatisfactory platelet count. The immediate postoperative outcome was good after the shunt. She did well and became alert with a GCS of 15 .

The absence of evidence-based guidelines for platelet transfusion surrounding invasive neurosurgical procedures leads to uncertainty in management. Multiple studies have illuminated this lack of high-quality data and subsequent reliance on expert opinion. The generally accepted threshold for platelet transfusion has hovered around $100,000 / \mu \mathrm{L}$ [11]. When to operate in this type of situation? The question remains unanswered. In fact, our patient presented eight hours later an acute right-hemispheric subdural hematoma for which she underwent surgery for the evacuation of the hematoma by craniectomy with a lethal outcome two weeks later despite the good evacuation of the hematoma. We considered the mechanism of this hematoma in relation to hyper drainage secondarily complicated by cortical detachment. However, it appears more likely that severe thrombocytopenia is sufficient to explain this complication. For Li et al. [11] the available evidence is sparse and of low quality but suggests that a platelet count $<$ $100,000 / \mu \mathrm{L}$ is associated with increased risk of hemorrhagic complications. In addition, the acuity of thrombocytopenia, magnitude of decrease in platelet count, and responsiveness to platelet transfusions impact the risk of neurosurgical intervention and should be taken into account when evaluating a patient's surgical candidacy.

\section{Conclusion}

Severe thrombocytopenia is a condition in which we have a low blood platelet less than $50.000 \mu \mathrm{L}$. It might occur as a result of a bone marrow disorder such as an immune system, leukemia, or can complicate the management of neurosurgery by significantly increasing the potential risk of bleeding during or after an invasive procedure. The treatment for severe thrombocytopenia is platelet transfusion.

\section{Disclosure}

The authors did not receive any funding for the preparation of this case report.

This article is an original work that is not being considered or reviewed by any other publication, and has not been published elsewhere in the same or a similar form.

All authors of the manuscript have read and agreed to its content and are ac- 
countable for all aspects of the accuracy and integrity of the manuscript.

\section{Informed Consent}

The patient gave his informed consent to publish his case.

\section{Authors' Contributions}

Yao Christian Hugues Dokponou: Conceptualization, writing original draft, \& editing. Fernand Nathan Imoumby: Writing, review \& editing. Imbunhe Napoléon: Review \& editing. Miloudi Gazzaz: Supervision, validation, \& review.

\section{Conflicts of Interest}

The authors declare that they have no conflicts of interest.

\section{References}

[1] Grant, G.A. (2007) Update on Hemostasis: Neurosurgery. Surgery, 142, S55-S60. https://doi.org/10.1016/j.surg.2007.06.030

[2] Glance, L.G., Blumberg, N., Eaton, M.P., Lustik, S.J., Osler, T.M., Wissler, R., et al. (2014) Preoperative Thrombocytopenia and Postoperative Outcomes after Noncardiac Surgery. Anesthesiology, 120, 62-75. https://doi.org/10.1097/ALN.0b013e3182a4441f

[3] Moore, E.W., Thomas, L.B., Shaw, R.K. and Freireich, E.J. (1960) The Central Nervous System in Acute Leukemia: A Postmortem Study of 117 Consecutive Cases, with Particular Reference to Hemorrhages, Leukemic Infiltrations, and the Syndrome of Meningeal Leukemia. AMA Archives of Internal Medicine, 105, 451-468. https://doi.org/10.1001/archinte.1960.00270150105011

[4] Nies, B.A., Thomas, L.B. and Freireich, E.J. (1965) Meningeal Leukemia. A Follow-Up Study. Cancer [Internet], 18, 546-553. https://acsjournals.onlinelibrary.wiley.com/doi/abs/10.1002/1097-0142\%28196505\% 2918\%3A5\%3C546\%3A\%3AAID-CNCR2820180503\%3E3.0.CO\%3B2-E

[5] Acute Myeloid Leukemia (AML) [Internet]. Aplastic Anemia \& MDS International Foundation. https://www.aamds.org/diseases/acute-myeloid-leukemia-aml

[6] Jameson-Lee, M., Chen, K., Ritchie, E., Shore, T., Al-Khattab, O. and Gergis, U. (2018) Acute Myeloid Leukemia in a Patient with Thrombocytopenia with Absent Radii: A Case Report and Review of the Literature. Hematology Oncology and Stem Cell Therapy, 11, 245-247. https://doi.org/10.1016/j.hemonc.2017.02.001

[7] Ogundeji, J.A., et al. (2021) Immune Thrombocytopenic Purpura Following Acute Lymphoblastic Leukemia in Remission. Journal of Leukemia, 3, 2. https://doi.org/10.4172/2329-6917.1000180

[8] Kvolik, S., Jukic, M., Matijevic, M., Marjanovic, K. and Glavas-Obrovac, L. (2010) An Overview of Coagulation Disorders in Cancer Patients. Surgical Oncology, 19, e33-46. https://doi.org/10.1016/j.suronc.2009.03.008

[9] Kitchens, C.S. (1994) Preoperative PTs, PTTs, Cost-Effectiveness, and Health Care Reform: Radical Changes That Make Good Sense. Chest, 106, 661-662.

https://doi.org/10.1378/chest.106.3.661

[10] Schramm, B., Leslie, K., Myles, P.S. and Hogan, C.J. (2001) Coagulation Studies in Preoperative Neurosurgical Patients. Anaesthesia and Intensive Care, 29, 388-392. https://doi.org/10.1177/0310057X0102900410 
[11] Li, D., Glor, T. and Jones, G.A. (2017) Thrombocytopenia and Neurosurgery: A Literature Review. World Neurosurgery, 106, 277-280.

https://doi.org/10.1016/j.wneu.2017.06.097 